\title{
Sen Speed: Sensing Driving Conditions to Estimate Vehicle Speed in Urban Environment
}

\author{
M. Naveen Kumar ${ }^{1}$, Dr. K. Anbarasan² \\ P.G Scholar, EST, Dhanalakshmi Srinivasan Engineering College, Perambalur, India ${ }^{1}$ \\ Professor and HOD / EEE, Dhanalakshmi Srinivasan Engineering College, Perambalur, India ${ }^{2}$
}

\begin{abstract}
Acquiring instant vehicle speed is desirable and a corner stone to many important vehicular applications. Ultrasonic Sensors to estimate the vehicle speed. We estimate the vehicle speed by Using Ultrasonic sensors. The obstacle avoidance vehicle is used for detecting obstacle and avoiding the collision. The design of obstacle Vehicle requires the integration of many sensors according to their task. Ultrasonic sensor is most suitable for obstacle detection and it is of low cost and has high ranging capability. Very small over time which can be corrected at some points, called reference points, where the true vehicle speed can be estimated. which senses natural driving conditions in urban environments including making turns, stopping and passing through uneven road surfaces, Extensive experiments demonstrate that Senspeed is accurate and robot in real driving environments. If the vehicle temperature high, the vehicle will be stopped. The ZIGBEE wireless module is received the targeted distance and it's monitoring from PC.
\end{abstract}

Index Terms: Ultrasonic Sensor, ZIGBEE, Estimate vehicle speed, Temperature sensor, gas sensor, DC motor, PIC controller.

\section{INTRODUCTION}

Zigbee based vehicular applications become more and more popular to analyze the increasingly complex urban traffic flows and facilitate more intelligent driving experiences including vehicle localization. Enhancing driving safety. The vehicle speed is an essential input. Accurate vehicle speed estimation could make those vehicle-speed dependent applications more reliable under complex traffic systems in urban environments. The project is designed to build an obstacle avoidance robotic vehicle using ultrasonic sensors for its movement. PIC is used to achieve the desired operation. Robotics is generally a combination of computational intelligence and physical machines (motors). Computational intelligence involves the programmed instructions. The project proposes robotic vehicle that has an intelligence built in it such that it guides itself whenever an obstacle comes ahead of it. This robotic vehicle is built, using a PIC. An ultrasonic sensor is used to detect any obstacle ahead of it and sends a command to the PIC.The Zigbee-based vehicular applications become more and more popular to analyze the increasingly complex urban traffic flows and facilitate more intelligent driving experiences including vehicle localization, enhancing driving safety, driving behavior analysis and building intelligent transportation systems. Among these applications, the vehicle speed is an essential input. Accurate vehicle speed estimation could make those vehicle-speed dependent applications more reliable under complex traffic systems in urban environments. In this paper we consider a sensing approach, which uses Ultrasonic and temperature sensors to sense natural driving conditions, to derive the vehicle speed without requiring any additional hardware. The basic idea is to obtain the vehicle's speed estimation by Ultrasonic sensors readings along the vehicle's moving direction over time. While the idea of integrating the acceleration values over time seems simple, a number of challenges arise in practice. The speed estimation should be real-time and accurate.

\section{II.ARCHITECTURE AND OPERATIONAL FRAMEWOR}

In this project proposed to envisioned that modern vehicles produced in the near future will be equipped with more wireless sensors, which are parts of the IVWSNs, to improve fuel economy, safety, engine performance, and offer more features. Based on the observation that some of these wireless sensors have low duty cycles, it is shown that additional features and functionalities can be provided by utilizing the idle time of these sensors. The problem of performing accurate vehicle speed estimation in urban environments to support pervasive vehicular applications. We employ Ultrasonic sensors to sense natural driving conditions to achieve high estimation accuracy. We propose a vehicle speed estimation system called Senspeed to identify three useful reference points, including making turns, vehicle stopping, and passing through uneven road surfaces. In this paper, the blind zone alert system is chosen as an illustrative application; a specially designed system is installed in the rear of a vehicle and it detects the presence of a target vehicle in its blind zone based on the received signal strength of packets broadcast by the sensors such as ultrasonic sensors of 
the target vehicle. This system is developing by PIC 16F877A and ZIGBEE module are always interfaced. In this proposed system also to provide automatic steering control at intruder detection. The ZIGBEE wireless module is received the targeted distance and it's monitoring from PC.

\section{A. BLOCK DIAGRAM}

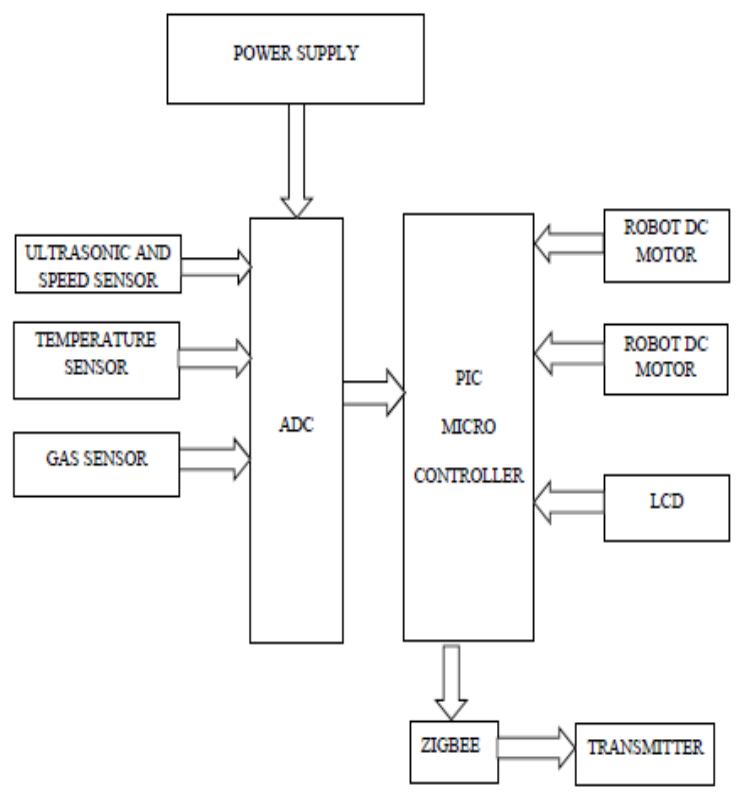

Figure: 1.Block diagram of transmitter

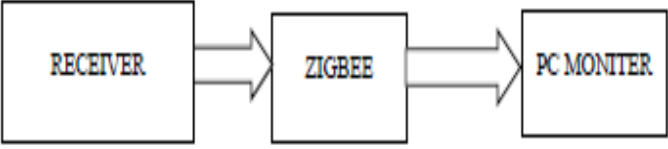

Figure:2.Block diagram of receiver

\section{B.PIC CONTROLLER}

The PIC controlled system often resides machines that are expected to run continuously for many years without any error and in some cases recover by themselves if an error occurs(with help of supporting firmware). Many of the PIC based embedded system use a simple pipelined RISC processor for computation and most of them provide on-chip SRAM for data storage to improve the performance. A PIC controlled system operates with minimal power consumption without sacrificing performance. Power consumption can be reduced by independently and dynamically controlling multiple power platforms. Most of the PIC based systems are memory expandable and will help in easily adding more and more memory according to the usage and type of application. In small applications the inbuilt memory can be used.PIC 16F877 is one of the most advanced microcontroller from Microchip. This controller is widely used for experimental and modern applications because of its low price, wide range of applications, high quality, and ease of availability. It is ideal for applications such as machine control applications, measurement devices, study purpose, and so on. The PIC 16F877 features all the components which modern microcontrollers normally have. The figure of a PIC16F877 chip is shown below. The basic building block of PIC 16F877 is based on Harvard architecture. This microcontroller also has many advanced features as mentioned in the previous post. Here you can see the basic internal architecture and memory organization of PIC16F877. This microcontroller has three types of memory- ROM, RAM and EEPROM.

\section{C.LIQUID CRYSTAL DISPLAY}

A liquid crystal display (LCD) is a flat panel display, electronic visual display, or video display that uses the light modulating properties of liquid crystals. Liquid crystals do not emit light directly. LCDs are available to display arbitrary images (as in a general-purpose computer display) or fixed images which can be displayed or hidden, such as preset words, digits, and 7-segment displays as in a digital clock. They use the same basic technology, except that arbitrary images are made up of a large number of small pixels, while other displays have larger elements. An LCD is a 


\section{ISO 3297:2007 Certified}

Vol. 5, Issue 5, May 2017

small low cost display. It is easy to interface with a micro-controller because of an embedded controller (the black blob on the back of the board).

\section{D.TEMPERATURE SENSOR (LM35)}

The LM35 is an integrated circuit sensor that can be used to measure temperature with an electrical output proportional to the temperature $\left(\right.$ in ${ }^{\circ} \mathrm{C}$ ). The LM35 generates a higher output voltage than thermocouples and may not require that the output voltage be amplified. The LM35 does not require any external calibration or trimming to provide typical accuracies of $\pm 1 / 4^{\circ} \mathrm{C}$ at room temperature and $\pm 3 / 4^{\circ} \mathrm{Cover}$ a full -55 to $+150^{\circ} \mathrm{C}$ temperature range. Low cost is assured by trimming and calibration at the wafer level.

\section{E.GAS SENSOR}

A gas detector is a device that detects the presence of gases in an area, often as part of a safety system. This type of equipment is used to detect a gas leak and interface with a control system so a process can be automatically shut down. A gas detector can sound an alarm to operators in the area where the leak is occurring, giving them the opportunity to leave. This type of device is important because there are many gases that can be harmful to organic life, such as humans or animals. Gas detectors can be used to detect combustible, flammable and toxic gases, and oxygen depletion. This type of device is used widely in industry and can be found in locations, such as on oil rigs, to monitor manufacture processes and emerging technologies such as photovoltaic. They may be used in firefighting. Gas leak detection is the process of identifying potentially hazardous gas leaks by sensors. These sensors usually employ an audible alarm to alert people when a dangerous gas has been detected. Common sensors include infrared point sensors, ultrasonic sensors, electrochemical gas sensors, and semiconductor sensors. More recently, infrared imaging sensors have come into use. All of these sensors are used for a wide range of applications and can be found in industrial plants, refineries, waste-water treatment facilities, vehicles, and homes.

\section{F.ULTRASONIC SENSOR}

Ultrasonic transducers are transducers that convert ultrasound waves to electrical signals or vice versa. Those that both transmit and receive may also be called ultrasound transceivers; many ultrasound sensors besides being sensors are indeed transceivers because they can both sense and transmit. These devices work on a principle similar to that of transducers used in radar and sonar systems, which evaluate attributes of a target by interpreting the echoes from radio or sound waves, respectively. Active ultrasonic sensors generate high-frequency sound waves and evaluate the echo which is received back by the sensor, measuring the time interval between sending the signal and receiving the echo to determine the distance to an object. Passive ultrasonic sensors are basically microphones that detect ultrasonic noise that is present under certain conditions, convert it to an electrical signal, and report it to a computer. This technology can be used for measuring wind speed and direction (anemometer), tank or channel fluid level, and speed through air or water. For measuring speed or direction, a device uses multiple detectors and calculates the speed from the relative distances to particulates in the air or water.

\section{G.L293D -DC MOTOR DRIVER IC}

We start with the L293D. L293D is a popular motor driving IC. It is a 16 pin IC. The IC has 8 pins on both the sides. It has 2 enable pins, $1 \mathrm{~V}_{\mathrm{ss}}$ pin, $1 \mathrm{~V}_{\mathrm{s}}$ pin, 4 ground pins, 4 input pins and 4 output pins. Though not required here, but in case you wish to learn how to microcontroller. The L293D IC can be used to control a maximum of 4 motors simultaneously. When 4 motors are connected to the IC, then for operation, Negative of each of the motors is connected to the GND, and the positive terminal to the outputs. For bidirectional control, you can connect only two motors simultaneously as per the circuit diagram below:

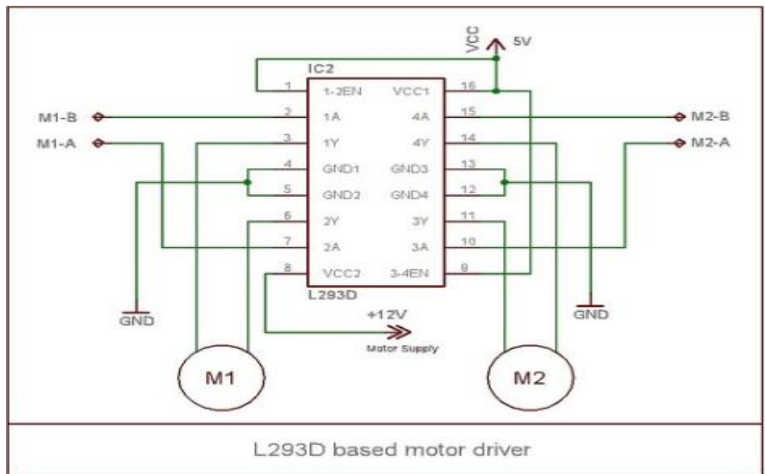

Figure: 3.Circuit diagram of dc motor driver 


\section{H.ZIGBEE TECHNOLOGY}

ZigBee is the global Wireless Technology connecting dramatically various devices to work together and enhance everyday life. ZigBee was introduced by IEEE and the ZigBee Alliance provides the standards for various applications. ZigBee is a low power, low cost, low data rate wireless standard for WPAN featured with security, reliability, large network capacity,easily deployed, short delay, long transmission range. There are three categories of nodes in a ZigBee system. They are Coordinator which is responsible for initiating the network and selecting the network parameters, Router which act as intermediate nodes, relaying data from other devices. End Devices can be low-power or battery powered devices. ZigBee coordinator node is completely responsible for the initialization, control and maintaining the network. Network may be extended by using ZigBee routers. This reduced functionality allows the device to reduce their cost. Star, Mesh \& Tree are the three topologies which support the ZigBee network. Every node in the network can transmit and receive data in its wireless rangeZigBee protocol stack defines four layer namely PHY layer, MAC layer, Network layer, Application layer. ZigBee defines Application \& Network layer whereas PHY \& MAC layer are defined by IEEE 802.15.4. Based on data processing capabilities, two types of devices are provided in IEEE 802.15.4 Full Function Device and Reduced Function Device Full Function Device support complete protocol function and can act as Coordinator, Router and End Device.

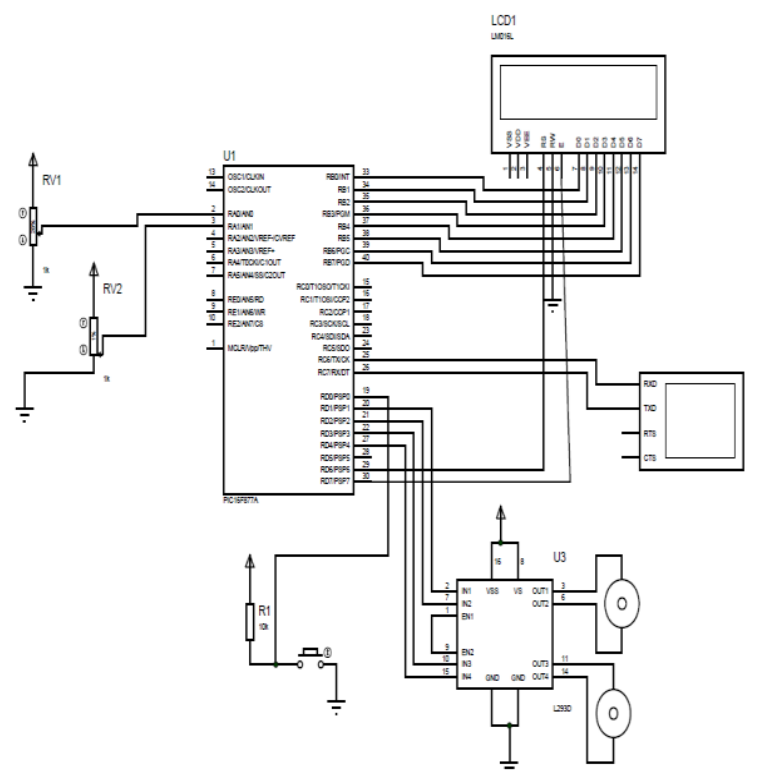

Figure: 4.Proposed circuit diagram

Ultrasonic transducers are transducers that convert ultrasound waves to electrical signals or vice versa. Those that both transmit and receive may also be called ultrasound transceivers; many ultrasound sensors besides being sensors are indeed transceivers because they can both sense and transmit. These devices work on a principle similar to that of transducers used in radar and sonar systems, which evaluate attributes of a target by interpreting the echoes from radio or sound waves, respectively. Passive ultrasonic sensors are basically microphones that detect ultrasonic noise that is present under certain conditions, convert it to an electrical signal, and report it to a computer.This technology can be used for measuring wind speed and direction (anemometer), This technology, as well, can detect approaching objects and track their positions.

LM35 temperature sensor can measure more accurately than using a thermistor. The LM35 generates a higher output voltage than thermocouples and may not require that the output voltage be amplified. Gas leak detection is the process of identifying potentially hazardous gas leaks by sensors. These sensors usually employ an audible alarm to alert people when a dangerous gas has been detected.Electrical DC Motors are continuous actuators that convert electrical energy into mechanical energy. The DC motor achieves this by producing a continuous angular rotation that can be used to rotate pumps, fans, compressors, wheels, etc., we employ Ultrasonic sensors to sense natural driving conditions to achieve high estimation accuracy. We propose a vehicle speed estimation system called Senspeed to identify three useful reference points, including making turns, vehicle stopping, and passing through uneven road surfaces. In this paper, the blind zone alert system is chosen as an illustrative application; a specially designed system is installed in the rear of a vehicle and it detects the presence of a target vehicle in its blind zone based on the received signal strength of packets broadcast by the sensors such as ultrasonic sensors of the target vehicle. 
International Journal of Innovative Research in Electrical, Electronics, Instrumentation and Control Engineering

ISO 3297:2007 Certified

Vol. 5, Issue 5, May 2017

\section{SIMULATION RESULT}

\section{A.MOTOR OFF:}

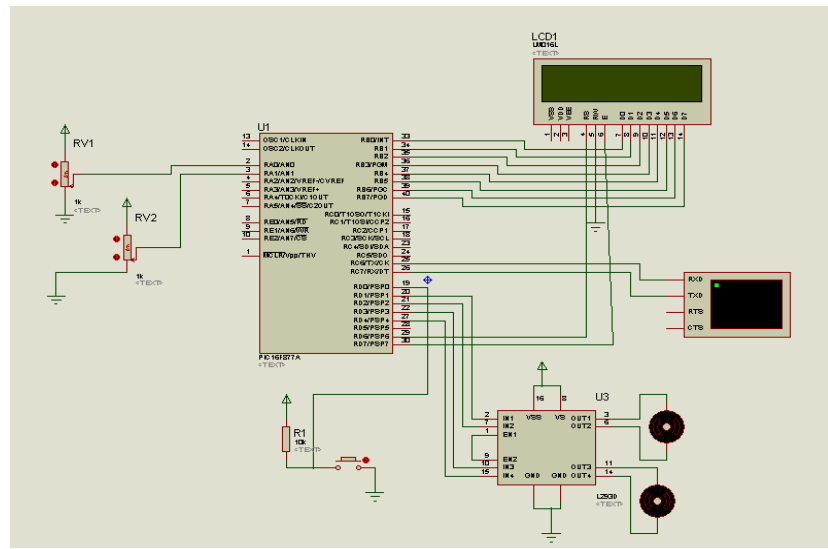

Figure: 5. Simulation Result For the motor off condition

\section{B.MOTOR ON:}

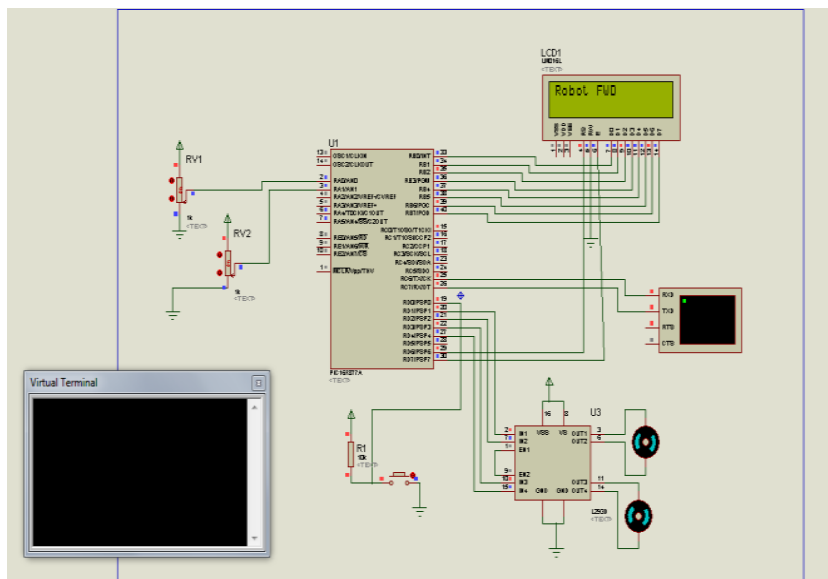

Figure: 6. Simulation Result For the motor on condition

\section{C.OBJECT IS DETECTED, MOTOR SPEED REDUCED:}

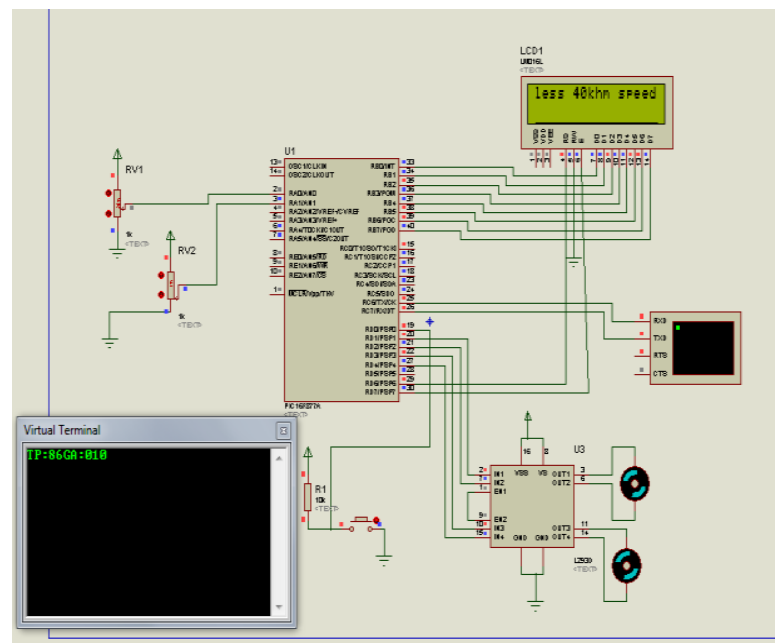

Figure: 7.Simulation Result for the Object detected, motor speed reduced 


\section{D.OBJECT IS NOT DETECTED:}

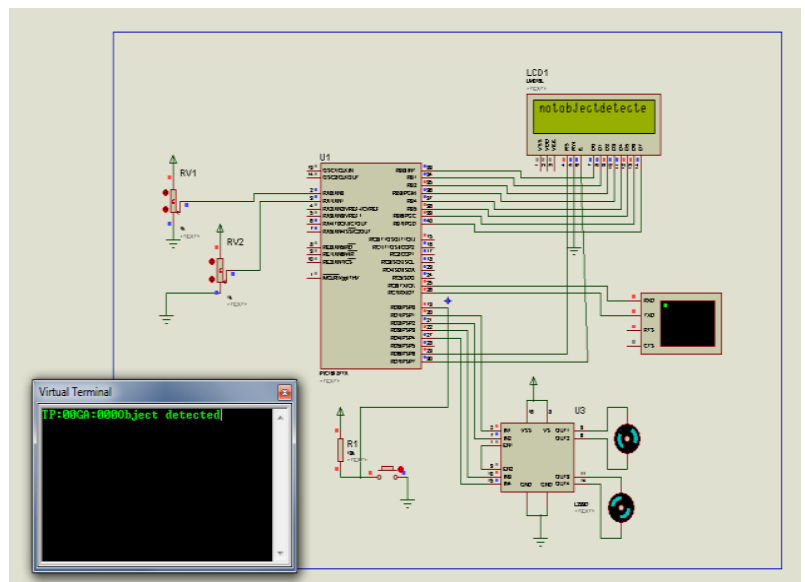

Figure: 8.Simulation Result for the Object is not detected

\section{CONCLUSION}

We address the problem of performing accurate vehicle speed estimation in urban environments to support pervasive vehicular applications. We employ smartphone sensors to sense natural driving conditions to achieve high estimation accuracy. In particular, we propose a vehicle speed estimation system called SenSpeed to identify three useful reference points, including making turns, vehicle stopping, and passing through uneven road surfaces, to measure and eliminate the errors caused by directly using phone's accelerometer readings for speed estimation. The key insight is that natural driving conditions present unique features and can be exploited to enable accurate real-time vehicle speed estimation. Our extensive experiments driving in two different cities over one month time period show that SenSpeed can estimate the vehicle speed in real-time with a low average error of $2.12 \mathrm{~km} / \mathrm{h}$, while achieving $1.21 \mathrm{~km} / \mathrm{h}$ during the offline estimation.

\section{REFERENCES}

[1] J. Levinson and S. Thrun, "Robust vehicle localization in urban environments using probabilistic maps," in Proc. IEEE Int. Conf. on Robotics and Automation, 2010, pp. 4372-4378.

[2] F. Chausse, J. Laneurit, and R. Chapuis, "Vehicle localization on a digital map using particles filtering," in Proc. Symp. Intelligent Vehicles, 2005, pp. 243-248.

[3] Y. Wang, J. Yang, H. Liu, Y. Chen, M. Gruteser, and R. P. Martin, "Sensing vehicle dynamics for determining driver phone use," in Proc. of ACM MobiSys'13, 2013, pp. 41-54.

[4] J. White, C. Thompson, H. Turner, B. Dougherty, and D. C. Schmidt, "Wreckwatch: Automatic traffic accident detection and notification with smartphones," Mob. Netw. Appl., vol. 16, no. 3, pp. 285-303, Jun. 2011

[5] J. Paefgen, F. Kehr, Y. Zhai, and F. Michahelles, "Driving behavior analysis with smartphones: insights from a controlled field study," in Proc. of ACM MUM'12, 2012, pp. 36:1-36:8.

[6] D. Johnson and M. Trivedi, "Driving style recognition using a smartphone as a sensor platform," in Proc. of IEEE Int. Conf. on Intelligent Transportation Systems, 2011, pp. 1609-1615.

[7] P. Mohan, V. N. Padmanabhan, and R. Ramjee, "Nericell: rich monitoring of road and traffic conditions using mobile smartphones," in Proc. of ACM SenSys'08, 2008, pp. 323-336.

[8] Schoepflin, "Dynamic camera calibration of roadside traffic management cameras for vehicle speed estimation," IEEE Trans. on Intelligent Transportation Systems, vol. 4, no. 2, pp. 90-98, 2003.

[9] A. Gorski. "understandinggps performance in urban environments". [Online]. Available: http://blogs.agi.com/agi/2011/ 01/04/understanding-gpsperformance-in-urban-environments/

[10] G. Chandrasekaran, T. Vu, A. Varshavsky, M. Gruteser, R. Martin, J. Yang, and Y. Chen, "Tracking vehicular speed variations by warping mobile phone signal strengths," in Proc. IEEE PerCom'2011, 2011, pp. 213-221.

[11] G. Chandrasekaran, T. Vu, A. Varshavsky, M. Gruteser, R. P. Martin, J. Yang, and Y. Chen, "Vehicular speed estimation using received signal strength from mobile phones," in Proc. ACM Ubicomp'10, 2010, pp. 237-240.

[12] B. Coifman, "Improved velocity estimation using single loop detectors," Transportation Research Part A: Policy and Practice, vol. 35, no. 10, pp. $863-880,2001$.

[13] B. Coifman, "Using dual loop speed traps to identify detector errors," Transportation Research Record: Journal of the Transportation Research Board, vol. 1683, pp. 47-58, 1999.

[14] V. Cevher, R. Chellappa, and J. McClellan, "Vehicle speed estimation using acoustic wave patterns,” IEEE Trans. on Sonignal Processing,, vol. 57, no. 1, pp. 30-47, 2009.

[15] V. Tyagi, S. Kalyanaraman, and R. Krishnapuram, "Vehicular traffic density state estimation based on cumulative road acoustics," IEEE Trans. on Intelligent Transportation Systems,, vol. 13-3, pp. 1156-1166, 2012. 